\title{
Comparison of Surgical Ligation and Endovascular Embolization in the Management of Type II Congenital Extrahepatic Portosystemic Shunt: A Retrospective Clinical Study
}

\section{Jin-long Zhang}

Department of Interventional Radiology, the First Medical Center of Chinese PLA General Hospital https://orcid.org/0000-0003-3158-2130

\section{Wei Dong Duan}

Department of Hepatobiliary Surgery, the First Medical Center of Chinese PLA General Hospital

\section{Zhu Ting Fang}

Shengli Clinical Medical College of Fujian Medical University

\section{Mao Qiang Wang}

Department of Interventional Radiology, the First Medical Center of Chinese PLA General Hospital Li Cui

Department of Interventional Radiology, the First Medical Center of Chinese PLA General Hospital

\section{Yan Hua Bai}

Department of Interventional Radiology, the First Medical Center of Chinese PLA Genral Hospital

\section{Xiao Hui Li}

Department of Interventional Radiology, the First Medical Center of Chinese PLA General Hospital

\section{Qi Cong Du}

Department of Radiology, the First Medical Center of Chinese PLA General Hospital

\section{Meng Qiu Shen}

Department of Interventional Radiology, the First Medical Center of Chinese PLA General Hospital

Feng Duan ( $\nabla$ duanfeng@vip.sina.com )

Department of Interventional Radiology, the First Medical Center of Chinese PLA General Hospital

\section{Research}

Keywords: Congenital extrahepatic portosystemic shunt, Abernethy malformation, Surgical ligation, Endovascular embolization, Portal vein

Posted Date: February 5th, 2021

DOl: https://doi.org/10.21203/rs.3.rs-184047/v1 
License: (c) (i) This work is licensed under a Creative Commons Attribution 4.0 International License. Read Full License 


\section{Abstract}

Background: Surgical ligation and endovascular embolization have been recommended for type II congenital extrahepatic portosystemic shunt (CEPS); however, no consensus has been reached. This study was designed to compare the safety and efficacy of surgical ligation and endovascular embolization for the treatment of type II CEPS.

Methods: In this retrospective study, 23 consecutive patients diagnosed with type II CEPS between March 2011 and April 2019 were divided into either a surgical group $(n=13 ; 41.5 \pm 19.9$ years) or the interventional group ( $n=10 ; 44.9 \pm 19.7$ years). The surgical group underwent laparoscopic surgical ligation of the shunt alone or ligation of the shunt and splenic artery and/or vein. The interventional group underwent endovascular embolization using microcoils, detachable coils and vascular plug.

Results: All 23 patients received a one-step shunt closure, and their clinical symptoms were significantly improved within 3 months post-procedure and without recurrence during follow-up. The serum ammonia levels in both groups decreased after the procedure and dropped to normal level at 6 to 12 months postprocedure. Compared with baseline, the portal vein diameter in interventional group increased significantly at 3-, 6-, 12-, and 36-month post-occlusion ( $\mathrm{P}=0.01$ for all). The procedure time was shorter in interventional group $(127.0 \pm 43.2$ minutes $)$ than surgical group $(219.8 \pm 56.7$ minutes; $P<0.001)$. The intraoperative blood loss in interventional group $(32.0 \pm 62.5 \mathrm{~mL})$ was less than that in surgical group (238.5 $\pm 396.9 \mathrm{~mL} ; \mathrm{P}=0.001)$.

Conclusion: Both surgical ligation and endovascular embolization are effective in the treatment of type II CEPS. Endovascular embolization has the advantages of shorter procedure time, and less intraoperative blood loss. The ligation of the portosystemic shunt and splenic artery and vein is feasible with apparent safety, and it could avoid a second surgical treatment.

\section{Background}

A congenital extrahepatic portosystemic shunt (CEPS), also known as an Abernethy malformation, is classified based on the extent of intact hypoplastic portal veins. Type I CEPS is characterized with an endto-side portocaval shunt and the absence of intrahepatic portal vein branches, while type II CEPS has some hypoplastic intrahepatic portal veins preserved with a side-to-side portocaval shunt diverting the portal vein blood to the inferior vena cava (IVC)[1-5]. Although CEPS is a rare clinical disease with less than 400 cases reported, it may develop severe complications, such as hepatic encephalopathy, pulmonary arterial hypertension, hepatopulmonary syndrome, or nodular liver lesions, among others[6].

To date, most of the published articles regarding CEPS are case reports, with a few small-sample clinical studies. Therefore, no consensus is achieved in the treatment of CEPS [6]. The recommended strategies for asymptomatic patients with CEPS are keeping them under careful observation or prophylactic interventional therapy[6]. Medical treatment is primarily used to improve symptoms caused by CEPS or its complications. While liver transplantation is the only curative treatment for type I CEPS, shunt occlusion 
by surgical ligation or endovascular embolization are the most common treatments for type II CEPS. The use of shunt occlusion depends on the expertise of local surgeons and the length and caliber of the shunts; however, a comparison of the two occlusion modalities in the management of type II CEPS has not been reported. We therefore retrospectively compared the safety and efficacy of surgical ligation and endovascular embolization in the treatment of type II CEPS from two medical centers.

\section{Methods}

\section{Study design and patient population}

This was a retrospective clinical study performed at two medical centers in accordance with the Declaration of Helsinki and approved by the respective institutional review boards. All patients provided written informed consent prior to treatment. All patients in this study have not been reported.

This retrospective study included consecutive patients diagnosed with CEPS through abdominal ultrasound, computed tomographic angiography (CTA) and/or magnetic resonance imaging (MRI) between March 2011 and April 2019 in two hospitals. Study patient selection was performed in conjunction with radiologists, hepatobiliary surgeons, and interventional radiologists. Patients diagnosed with type II CEPS who presented with one malformation and at least one apparent CEPS manifestation or complication, including hepatic encephalopathy, gastrointestinal bleeding, pulmonary hypertension, hepatic myelopathy, hepatopulmonary syndrome, hepatic cirrhosis, or hepatic adenoma were included in the study. Patients with type I CEPS, asymptomatic patients, multiple malformations, hepatocellular carcinoma, coagulopathies, active infection, chronic renal failure, or contraindications to angiography and surgery were excluded.

Initially, portal venography by way of systemic venous access and traversal of the portosystemic shunt and a 15-min shunt balloon occlusion test were performed. Patients with a large and short shunt, which may have high risk of embolic materials migration, or portal venous pressure (PVP) $>25 \mathrm{mmHg}$ after test occlusion, underwent surgical ligation (surgical group), with all others undergoing endovascular embolization (interventional group).

\section{Portographyand shunt balloon occlusion test}

To evaluate hemodynamics, measure PVP, and assess whether the shunt vessel could be closed, Portal venography and shunt balloon occlusion test were conducted. However, considering the heterogeneous morphology of CEPS, a standard portogram does not exist. Each angiographic examination was tailored to the patients according to the prior image findings of the suspected CEPS.

In general, the right femoral artery and femoral vein were cannulated using a 4-F vascular sheath (Radifocus; Terumo, Tokyo, Japan) and 6 to 10-F vascular sheath (Radifocus Introducer Il; Terumo, Tokyo, Japan) using the Seldinger technique under local anesthesia or a transjugular or percutaneous transhepatic approach if the transfemoral vein approach failed. An indirect portal venography was then 
performed via selective angiography of the celiac artery, splenic artery, and superior mesenteric artery using a 4-F hepatic artery catheter (Cordis, Miami Lakes, FL, USA) inserted through the arterial vascular sheath. Next, direct venography of the inferior mesenteric vein, splenic vein, and portal vein were performed using a 4-F Cobra (Cordis) catheter inserted through the venous vascular sheath and catheterized into the portal vein. Lastly, a balloon occlusion test was performed (Fogarty, Thru Embolectomy Catheter, Edwards Lifesciences LLC, Irvine, CA, USA; Boston Scientific, Watertown, Mass) in the infrahepatic segment of IVC or the shunt to detect the intrahepatic portal branches and measure PVP before and $15 \mathrm{~min}$ after balloon occlusion. If the shunt was suitable for endovascular embolization and endovascular occlusion of the shunt was performed at the same time.

\section{Laparoscopic surgical ligation}

Under general anesthesia, a catheter was placed in the portal vein via the shunt through the femoral vein to monitor PVP. A $12 \mathrm{~mm}$ port for optics was placed in the umbilical area and three $5 \mathrm{~mm}$ working ports were placed in upper abdomen, right lower abdomen, and right lateral abdomen.

If PVP was $<25 \mathrm{mmHg}$ after $15 \mathrm{~min}$ of occlusion, without signs of intestinal wall edema and redness examined at laparoscopy, the shunt was ligated alone. If PVP was $>25 \mathrm{mmHg}$ and/or there were signs of intestinal wall edema and redness, the splenic artery and vein were dissected and test ligated if PVP was $<25 \mathrm{mmHg}$ without signs of intestinal wall edema and redness after $15 \mathrm{~min}$, the splenic artery and/or vein were also taped. Otherwise, partial occlusion of the shunt was performed and completely occluded during the follow-up, completing a so-called "two-step closure."

\section{Endovascular shunt occlusion}

The embolization materials used were either one, or a combination of 0.018 inch pushable micro coils (MicroNester; Cook Medical, Bloomington, USA), a 0.018 inch detachable coils (Interlock; Boston Scientific, Marlborough, Massachusetts, USA), and an Amplatzer vascular plug (16-mm, $18 \mathrm{~mm}, 20 \mathrm{~mm}$, or $22 \mathrm{~mm}$ AVP IIA; Abbott Park, Illinois囚USA). After selective shunt catheterization, the microcoils and interlock coil were inserted through a coaxial 2.7-F microcatheter (Progreat; Terumo, Tokyo, Japan) , the plug was inserted through a 6 to 10-F long sheath (Cook medical, USA). Indirect portography through SMA to detect whether there were residual shunts.

\section{Postprocedural treatment}

To prevent portal and mesenteric thrombosis, $30 \mathrm{mg}$ of subcutaneous enoxaparin was administered every $12 \mathrm{~h}$, beginning $12 \mathrm{~h}$ after the procedure and throughout 7 days of in-hospital observation. Patients were then discharged if no complications occurred and $10 \mathrm{mg}$ of oral rivaroxaban was administered once daily for 30 days.

Abdominal ultrasound and contrast-enhanced CT performed at 1 week and 1 month after the procedure determined whether portal vein system thrombosis had occurred. If there was an absence of thrombosis 1 month after the procedure, rivaroxaban was stopped. 


\section{Follow-up}

Follow-up at 3, 6, and 12 months after the procedure, and every 12 months thereafter included clinical status, routine blood tests, serum ammonia, liver function, coagulation, and abdominal contrastenhanced CT. The diameter of the main portal vein was measured by two radiologists independently, with the mean value used as the final result.

\section{Statistical analysis}

Continuous variables were recorded as means \pm standard deviations, and categorical data as numbers and percentages. All data were tested for normality using a Shapiro-Wilk test. Differences in PVP, hemoglobin, serum ammonia, portal vein diameter, and Child-Pugh score pre- and post-procedure within each group were compared with paired-Student $t$ test (normally distributed) or paired-Wilcoxon rank-sum test (not normally distributed). Differences between groups were tested using a Student $t$ test or Wilcoxon rank-sum test. Differences in the main symptoms, comorbidities, and location of shunt vessels between groups were tested using the two-tailed Fisher exact test. $P<0.05$ indicated a statistically significant difference (SPSS Statistics for Windows, version 20.0; IBM, Armonk, NY).

\section{Results}

\section{Patient characteristics}

Baseline demographic and clinical data of the study patients are presented in Table 1. A total of 27 patients were recruited, four cases were excluded due to type I CEPS $(n=1)$, heptocellular carcinoma $(n=$ $1)$, and incomplete data $(n=2)$. Twenty-three patients were included in the final analysis, 13 patients (five male, eight female; mean age, 40.7 years \pm 20.4 ; age range, $3-67$ years) in the surgical group and 10 patients (eight male, two female; mean age, 44.9 years \pm 19.7 ; age range, $17-69$ years) in the interventional group (Fig. 1). 
Table 1

Baseline Characteristics of Patients in Both Groups

\begin{tabular}{|c|c|c|c|}
\hline Characteristic & Surgical group $(n=13)$ & $\begin{array}{l}\text { Interventional group } \\
(n=10)\end{array}$ & $P$ Value \\
\hline Age & $40.7 \pm 20.4$ & $44.9 \pm 19.7$ & 0.68 \\
\hline Male & $5(38.5)$ & $8(80.0)$ & \\
\hline Female & $8(61.5$ & $2(20.0)$ & \\
\hline \multicolumn{4}{|l|}{ CEPS Symptoms } \\
\hline Hepatic encephalopathy & $6(46.2)$ & $5(50.0)$ & $>0.99$ \\
\hline Gastrointestinal bleeding & $4(30.8)$ & $2(20.0)$ & 0.66 \\
\hline Dyspnoea & $1(7.7)$ & $0(0)$ & $>0.99$ \\
\hline Abdominal pain & $1(7.7)$ & $1(10.0)$ & $>0.99$ \\
\hline Hepatic myelopathy & $1(7.7)$ & $1(10.0)$ & $>0.99$ \\
\hline Hemoptysis & $0(0)$ & $1(10.0)$ & $>0.99$ \\
\hline Fatigue & $2(15.4)$ & $1(10.0)$ & $>0.99$ \\
\hline \multicolumn{4}{|l|}{ Comorbidity } \\
\hline Hepatic cirrhosis & $10(76.9)$ & $5(50.0)$ & 0.22 \\
\hline Hepatic adenoma & $2(15.4)$ & $0(0)$ & 0.49 \\
\hline Pulmonary hypertension & $1(7.7)$ & $1(10.0)$ & $>0.99$ \\
\hline Hypersplenism & $1(7.7)$ & $1(10.0)$ & $>0.99$ \\
\hline \multicolumn{4}{|l|}{ Location of shunt vessels } \\
\hline Splenorenal shunt & $5(38.5)$ & $3(30.0)$ & $>0.99$ \\
\hline SMV-IVC shunt & $3(23.1)$ & $1(10.0)$ & 0.60 \\
\hline Portal vein-IVC shunt & $3(23.1)$ & $2(20.0)$ & $>0.99$ \\
\hline SMV-renal vein shunt & $1(7.7)$ & $1(10.0)$ & $>0.99$ \\
\hline Portal vein-renal vein shunt & $1(7.7)$ & $1(10.0)$ & $>0.99$ \\
\hline Portal vein-iliac vein shunt & $0(0)$ & $2(20.0)$ & 0.18 \\
\hline
\end{tabular}


The main manifestations of CEPS in this cohort were hepatic encephalopathy (six in the surgical group and five in the interventional group) and gastrointestinal bleeding (four in the surgical group and two in the interventional group) with no significant difference between groups $(P>0.99$ and $P=0.66$, respectively). Other manifestations included dyspnea, abdominal pain, hepatic myelopathy, hemoptysis, and fatigue, with no significant differences between groups $(P>0.99$ for all).

Of the 13 patients in the surgical group, 10 experienced hepatic cirrhosis, two experienced hepatic adenoma, one experienced pulmonary hypertension, and one experienced hypersplenism. Similarly, of the 10 patients in the interventional group, five experienced hepatic cirrhosis, one experienced pulmonary hypertension, and one experienced hypersplenism.

\section{Location Of Shunt Vessels}

In the surgical group, the types of shunt observed were splenorenal shunt $(n=5)$, superior mesenteric vein-inferior vena cava shunt (SMV-IVC; $n=3)$, portal vein-IVC shunt $(n=3), S M V$-renal vein shunt $(n=1)$, and portal vein-renal vein shunt $(n=1)$. While in the interventional group, splenorenal shunt $(n=3)$, SMVIVC $(n=1)$, portal vein-IVC shunt $(n=2)$, SMV-renal vein shunt $(n=1)$, portal vein-renal vein shunt $(n=1)$ and portal vein-iliac vein shunt $(n=2)$ were observed (Table 1; Figs. $2-3)$.

\section{Intraoperative Parameters}

All 23 patients received a one-step closure. For patients in the surgical group, three underwent shunt ligation alone, six underwent shunt and splenic artery ligation, and four patients underwent ligation of the shunt, and splenic artery and vein. The post-procedure PVP $(17.5 \pm 3.8 \mathrm{mmHg}$; range: $8.1-22.1 \mathrm{mmHg})$ was significantly greater than pre-procedure $(12.9 \pm 3.7 \mathrm{mmHg}$; range: $5.9-17.6 \mathrm{mmHg} ; P<0.001)$ in surgical group, but it was lower than $25 \mathrm{mmHg}$. In interventional group, the shunts were embolized using an Amplatzer vascular plug in four patients, with the remaining six cases using a combination of detachable and pushable coils (Figs. 2-3). The PVP were not significantly different pre- and postocclusion (Pre: $15.9 \pm 4.7 \mathrm{mmHg}$; range: $11.4-21.7 \mathrm{mmHg}$; Post: $17.1 \pm 4.8 \mathrm{mmHg}$; range: $13.2-24.3$ $\mathrm{mmHg} ; P=0.22$ ) (Table 2).

Table 2

Comparison of Portal Vein Pressure in Surgical and Interventional Groups Pre- and Post-procedure

\begin{tabular}{|llll|}
\hline Variable & Surgical group $(\mathrm{n}=13)$ & Interventional group $(\mathrm{n}=10)$ & $P$ Value \\
\hline Portal vein pressure $(\mathrm{mmHg})$ & & & \\
\hline Pre-procedure & $12.9 \pm 3.7$ & $15.9 \pm 4.7$ & 0.18 \\
\hline Post-procedure & $17.5 \pm 3.8$ & $17.1 \pm 4.8$ & 0.86 \\
\hline P Value & $<0.001$ & 0.22 & \\
\hline
\end{tabular}


The procedure time was significantly shorter in the interventional group $(127.0 \pm 43.2 \mathrm{~min})$ than that in surgical group $(219.8 \pm 56.7 \mathrm{~min} ; P<0.001)$. The intraoperative blood loss in the interventional group $(32.0 \pm 62.5 \mathrm{~mL})$ was significantly less than that of the surgical group $(238.5 \pm 396.9 \mathrm{~mL} ; \mathrm{P}=0.001)$. Additionally, the treatment expense of interventional group (46331.5 \pm 18839.1 Yuan) was lower than that of surgical group (59561.3 \pm 21450.1 Yuan) but without statistical significance $(P=0.20)$ (Table 3 ).

Table 3

Differences in Intraoperative Parameters between Surgical and Interventional Groups

\begin{tabular}{|llll|}
\hline Parameters & Surgical group $(\mathrm{n}=13)$ & Interventional group $(\mathrm{n}=10)$ & $P$ Value \\
\hline Procedure time $(\mathrm{min})$ & $219.8 \pm 56.7$ & $127.0 \pm 43.2$ & $<0.001$ \\
\hline Intraoperative blood loss $(\mathrm{mL})$ & $238.5 \pm 396.9$ & $32.0 \pm 62.5$ & 0.001 \\
\hline Treatment expense (Yuan) & $59561.3 \pm 21450.1$ & $46331.5 \pm 18839.1$ & 0.20 \\
\hline
\end{tabular}

\section{Clinical Outcomes}

The mean follow-up period was $26.1 \pm 17.6$ months (range, 6-60 months). Clinical symptoms of all patients significantly improved within 3 months of their procedure without recurrence during follow-up. Serum ammonia levels decreased after both procedures to normal levels $(9-30 \mu \mathrm{mol} / \mathrm{L})$ at 6 to 12 months post-procedure. The symptoms of hepatic encephalopathy and hepatic myelopathy associated with hyperammonemia were improved within 1 month of the procedure, and remained improved during the follow-up period (Table 4). 
Table 4

Clinical Outcomes in Surgical and Interventional Groups Pre- and Post-procedure

\begin{tabular}{|c|c|c|c|}
\hline Variable & Surgical group $(n=13)$ & Interventional group $(n=10)$ & $P$ Value \\
\hline \multicolumn{4}{|c|}{ Hemoglobin $(g / L)$} \\
\hline Pre-procedure & $109.4 \pm 26.2$ & $102.0 \pm 25.2$ & 0.46 \\
\hline 3-month & $104.0 \pm 27.0$ & $112.4 \pm 21.1$ & 0.08 \\
\hline 6-month & $114.0 \pm 8.5$ & $125.0 \pm 18.4$ & $<0.001$ \\
\hline 12-month & $104.3 \pm 19.8$ & $118.8 \pm 18.9$ & $<0.001$ \\
\hline 36-month & $125.5 \pm 24.7$ & $127.7 \pm 22.5$ & 0.03 \\
\hline \multicolumn{4}{|c|}{ Blood ammonia( $(\mu \mathrm{mol} / \mathrm{L})$} \\
\hline Pre-procedure & $83.6 \pm 32.5$ & $75.1 \pm 30.5$ & 0.12 \\
\hline 3-month & $56.4 \pm 20.8$ & $47.8 \pm 46.4$ & 0.003 \\
\hline 6-month & $41.0 \pm 1.4$ & $51.4 \pm 49.8$ & 0.01 \\
\hline 12-month & $19.2 \pm 6.8$ & $13.5 \pm 6.4$ & $<0.001$ \\
\hline 36-month & $24.0 \pm 6.4$ & $16.7 \pm 6.2$ & $<0.001$ \\
\hline \multicolumn{4}{|c|}{ Portal vein diameter (mm) } \\
\hline Pre-procedure & $8.5 \pm 4.9$ & $9.3 \pm 4.0$ & 0.25 \\
\hline 3-month & $9.6 \pm 3.4$ & $10.7 \pm 2.7$ & 0.34 \\
\hline 6-month & $9.7 \pm 3.0$ & $10.8 \pm 2.6$ & 0.22 \\
\hline 12-month & $10.0 \pm 3.0$ & $11.2 \pm 2.9$ & 0.09 \\
\hline 36-month & $10.2 \pm 2.9$ & $11.6 \pm 2.3$ & 0.046 \\
\hline \multicolumn{4}{|c|}{ Child-Pugh score } \\
\hline Pre-procedure & $7.8 \pm 1.7$ & $7.3 \pm 2.4$ & 0.36 \\
\hline 3-month & $6.7 \pm 1.9$ & $5.8 \pm 1.3$ & 0.01 \\
\hline 6-month & $5.5 \pm 0.7$ & $6.0 \pm 1.4$ & 0.001 \\
\hline 12-month & $6.0 \pm 1.4$ & $5.2 \pm 0.4$ & $<0.001$ \\
\hline 36-month & $5.5 \pm 0.7$ & $5.3 \pm 0.6$ & 0.001 \\
\hline
\end{tabular}

Patients with gastrointestinal bleeding (four in the surgical group and two in the interventional group) stopped bleeding after their procedure. One patient in the surgical group suffered hematemesis 6 months after occlusion. Gastroscopy revealed hemorrhage of a gastric ulcer, and bleeding stopped after 
endoscopic and acid suppression therapy. One patient with hemoptysis and one with dyspnea related to pulmonary hypertension achieved symptomatic relief at 1 week and 3 weeks after the procedure, respectively. Finally, one patient with hepatic adenoma underwent simultaneous resection.

Compared with baseline, the portal vein diameter in interventional group increased significantly at 3-, 6-, 12 -, and 36-month post-occlusion ( $P=0.01$ for all), but there was no significant difference in surgical group ( $P>0.05$ for all) (Table 4; Fig. 4).

Portal vein thrombosis was detected in one patient in the interventional group using contrast-enhanced CT scan at 1 month after the procedure. Rivaroxaban was discontinued, intravenous heparin sodium (40 $\mathrm{U} / \mathrm{kg}$ to control the activated partial thromboplastin time between 40 and $60 \mathrm{~s})$ and urokinase $(4000 \mathrm{U} / \mathrm{kg}$, twice daily) were administered. Seven days later, the portal vein thrombosis disappeared. Once daily oral rivaroxaban (10 mg) resumed for 4 months without thrombosis (Fig. 3).

\section{Complications}

No signs of portal hypertension and splenic abscess have been observed, and no other complications occurred.

\section{Discussion}

CEPS is a congenital malformation created by an abnormal shunt between the portal vein and vena cava or azygos/hemiazygos system due to abnormal development of the umbilical vein and yolk vein during the embryonic period. In combination with other congenital malformations, patients with CEPS present with hypergalactosemia, high bile acid, high serum ammonia, and hepatic encephalopathy caused by the portal and vena cava shunt. It has been reported that $66-100 \%$ of the patients with CEPS have hyperammonemia and $17-30 \%$ have hepatic encephalopathy[7-9].

Currently, liver transplantation is the only curative treatment for type I CEPS. Surgical ligation and endovascular embolization have been recommended to treat symptomatic type II CEPS; however, no consensus has been achieved and no comparison between the two procedures has been reported. Except for patients who are not suitable for endovascular occlusion due to shunt anatomy, no research has compared the more advantageous modality for patients suitable for both the treatment procedures so far. Our study found that both of the procedures were safe and effective in patients with type II CEPS. However, compared with laparoscopic surgical ligation, the endovascular approach has the advantages of shorter procedure time, less intraoperative blood loss, and lower treatment expense.

Angiography is the gold standard for diagnosing CEPS and identifying the type of CEPS present[10, 11]. While shunt anatomy and flow dynamics can be determined by angiography and/or direct venography, balloon shunt occlusion venography is critical for detecting intrahepatic portal vein branches. This 
branch identification could also determine the type of CEPS, and the pressure gradients pre- and postocclusion that is crucial for assessing the risk of portal hypertension after shunt occlusion[11-14].

In previous reports, $25 \mathrm{mmHg}$ was used as a threshold to monitor PVP. If PVP was less than $25 \mathrm{mmHg}$ after 15-20 min of occlusion, it indicated that the risk of portal hypertension was insignificant after shunt occlusion. Otherwise, a two-step closure is recommended with partial occlusion to initially acclimatize the intrahepatic portal system to increase flow and then completely occlude in 6 to 12 months[11,15-18].

In order to avoid portal hypertension post-occlusion for patients with PVP of more than $25 \mathrm{mmHg}$, we performed surgical ligation of the shunt combined with splenic artery and/or vein and thereby avoided two surgical procedures for shunt closing. Based on the abundant communication among the splenic artery, splenic vein, and splanchnic vessels, ligation of the splenic artery and splenic vein will not typically cause splenic necrosis or abscess. In this cohort, six patients underwent shunt and splenic artery ligation and four underwent additional splenic vein ligation. No portal hypertension or splenic abscess occurred and a second surgical treatment was avoided.

Portal vein and mesenteric venous thrombosis is a common complication after shunt ligation that can lead to portal hypertension; therefore, postprocedural anticoagulation therapy is very important $[6,10]$. Heparin and warfarin have been used to prevent thrombosis in previous studies; however, the activated partial thromboplastin time and international normalized ratio should be closely monitored to adjust drug dosage[16,19]. In our practice, enoxaparin and rivaroxaban have been used sequentially for anticoagulation after occlusion, and do not require monitoring indicators of coagulation and is more convenient to use. In the follow-up period, only one patient (1/23) suffered portal vein thrombosis, which disappeared after 7 days of conservative treatment.

Theoretically, the hypoplastic intrahepatic portal vein should dilate after the perfusion of portal vein blood increased due to shunt ligation. In this cohort, patients after endovascular embolization achieved significant increase in portal vein diameter, however, there was no significant difference in surgical ligation. This maybe attribute to the limited number of cases and the well-grow intrahepatic portal vein branches in several patients before occlusion.

Our study had some limitations. First, this study included only a small number of patients primarily due to the low incidence of CEPS. Second, the follow-up period was short, and due to the retrospective characteristics of the study, the follow-up intervals were not completely consistent and partial follow-up data was missing. Thirdly, the portosystemic gradient gradient was not measured.

\section{Conclusions}

Both surgical ligation and endovascular embolization are effective in the treatment of type II CEPS. The endovascular approach has the advantages of shorter procedure time, less intraoperative blood loss, and lower treatment expense. Surgical ligation is recommended for patients with a high risk of embolic materials migration due to short and large extrahepatic portosystemic shunt, and patients with PVP more 
than $25 \mathrm{mmHg}$ after test occlusion, which could lead to portal hypertension. Ligation of the portosystemic shunt and the splenic artery and vein is feasible with apparent safety, and it could avoid a second surgical treatment.

\section{Abbreviations}

CEPS: Congenital extrahepatic portosystemic shunt; CTA: Computed tomographic angiography; IVC: Inferior vena cava; MRI: Magnetic resonance imaging; PVP: Portal venous pressure; SMV: Superior mesenteric vein; SMA: Superior mesenteric artery

\section{Declarations}

\section{Acknowledgements}

Not applicable.

\section{Authors' contributions}

Study concept and design: F,D.; Acquisition of data: J.L.Z., W.D.D., Z.T.F., L.C., Y.H.B., X.H.L., M.Q.S.; Analysis and interpretation of data: J.L.Z., M.Q.W., F.D.; Image reconstruction: M.Q.W., Q.C.D. Drafting of the manuscript: J.L.Z., F.D.; Critical revision of the manuscript for important intellectual content: All authors; Statistical analysis: L.C., Y.H.B.; Study supervision: F.D. All authors read and approved the final manuscript.

\section{Funding}

This study was supported by grants from the Major National Science and Technology Projects (2020ZX1001010), the Beijing Talents Fund (2016000021223ZK25), the China Postdoctoral Science Foundation (2019M664006), and Natural Science Foundation of Fujian Province (202J011096).

\section{Availability of data and materials}

Not applicable.

\section{Ethics approval and consent to participate}

This study was in accordance with the Declaration of Helsinki and approved by the respective institutional review boards. All patients provided written informed consent prior to treatment.

\section{Consent for publication}

Not applicable.

\section{Competing interests}


The authors declare that there are no competing interests.

\section{References}

1. Morgan G, Superina R. Congenital absence of the portal vein: two cases and a proposed classification system for portasystemic vascular anomalies. J Pediatr Surg. 1994;29:1239-41.

2. Passalacqua M, Lie KT, Yarmohammadi H. Congenital extrahepatic portosystemic shunt (Abernethy malformation) treated endovascularly with vascular plug shunt closure. Pediatr Surg Int. 2012;28:79-83.

3. David A Jr, Meyer J, Archambeaud I, Frampas É, Perret C, Douane F. Endovascular Closure of a Large Congenital Extrahepatic Portosystemic Shunt (Abernethy Malformation) Using Vena Cava Filter and AMPLATZER Vascular Plugs. J Vasc Interv Radiol. 2018;29:1631-3.

4. Murray CP, Yoo SJ, Babyn PS. Congenital extrahepatic portosystemic shunts. Pediatr Radiol. 2003;33:614-20.

5. Stringer MD. The clinical anatomy of congenital portosystemic venous shunts. Clin Anat 2008; 21:147-157.6. Baiges A, Turon F, Simón-Talero M, et al. Congenital Extrahepatic Portosystemic Shunts (Abernethy Malformation): An International Observational Study. Hepatology 2020; 71:658669.

6. Lautz TB, Tantemsapya N, Rowell E, Superina RA. Management and classification of type II congenital portosystemic shunts. J Pediatr Surg. 2011;46:308-14.

7. Franchi-Abella S, Branchereau S, Lambert V, et al. Complications of congenital portosystemic shunts in children: therapeutic options and outcomes. J Pediatr Gastroenterol Nutr. 2010;51:322-30.

8. Kim MJ, Ko JS, Seo JK, et al. Clinical features of congenital portosystemic shunt in children. Eur J Pediatr. 2012;171:395-400.

9. Zhang B, Wu KT, Li L, Lai HY. Catheter Embolization of Type II Congenital Extrahepatic Portosystemic Shunt with Hematochezia: A Case Series and Review of the Literature. Cardiovasc Intervent Radiol. 2018;41:1121-7.

10. Mathai SV, Kondray V, Salloum E, Kukreja K, Tavri S. Role of interventional radiology in the diagnosis and management of congenital extrahepatic portosystemic shunts: Two case reports. Indian J Radiol Imaging. 2019;29:219-22.

11. Bernard O, Franchi-Abella S, Branchereau S, Pariente D, Gauthier F, Jacquemin E. Congenital portosystemic shunts in children: recognition, evaluation, and management. Semin Liver Dis. 2012;32:273-87.

12. Shah A, Aziz A, Awwad A, Ramjas G, Higashi Y. Incidental radiological diagnosis of asymptomatic Abernethy malformations-two case reports. BJR Case Rep. 2017;3:20150496.

13. Timpanaro T, Passanisi S, Sauna A, et al. Congenital portosystemic shunt: our experience. Case Rep Pediatr 2015; 2015:691618. 
14. Takama Y, Ueno T, Umeda S, Saka R, Tazuke Y, Okuyama H. Laparoscopic ligation of a congenital extrahepatic portosystemic shunt for children with hyperammonemia: a single-institution experience. Surg Today. 2019;49:323-7.

15. Mori T, Yamada Y, Abe K, et al. Laparoscopic Partial Closure for Congenital Portosystemic ShuntIndications, Postoperative Management, and Subsequent Complete Closure. J Laparoendosc Adv Surg Tech A. 2019;29:573-8.

16. Matsuura T, Takahashi Y, Yanagi Y, et al. Surgical strategy according to the anatomical types of congenital portosystemic shunts in children. J Pediatr Surg. 2016;51:2099-104.

17. Knirsch W, Benz DC, Bühr P, et al. Catheter interventional treatment of congenital portosystemic venous shunts in childhood. Catheter Cardiovasc Interv. 2016;87:1281-92.

18. Chocarro G, Amesty MV, Encinas JL, et al. Congenital Portosystemic Shunts: Clinic Heterogeneity Requires an Individual Management of the Patient. Eur J Pediatr Surg. 2016;26:74-80.

\section{Figures}

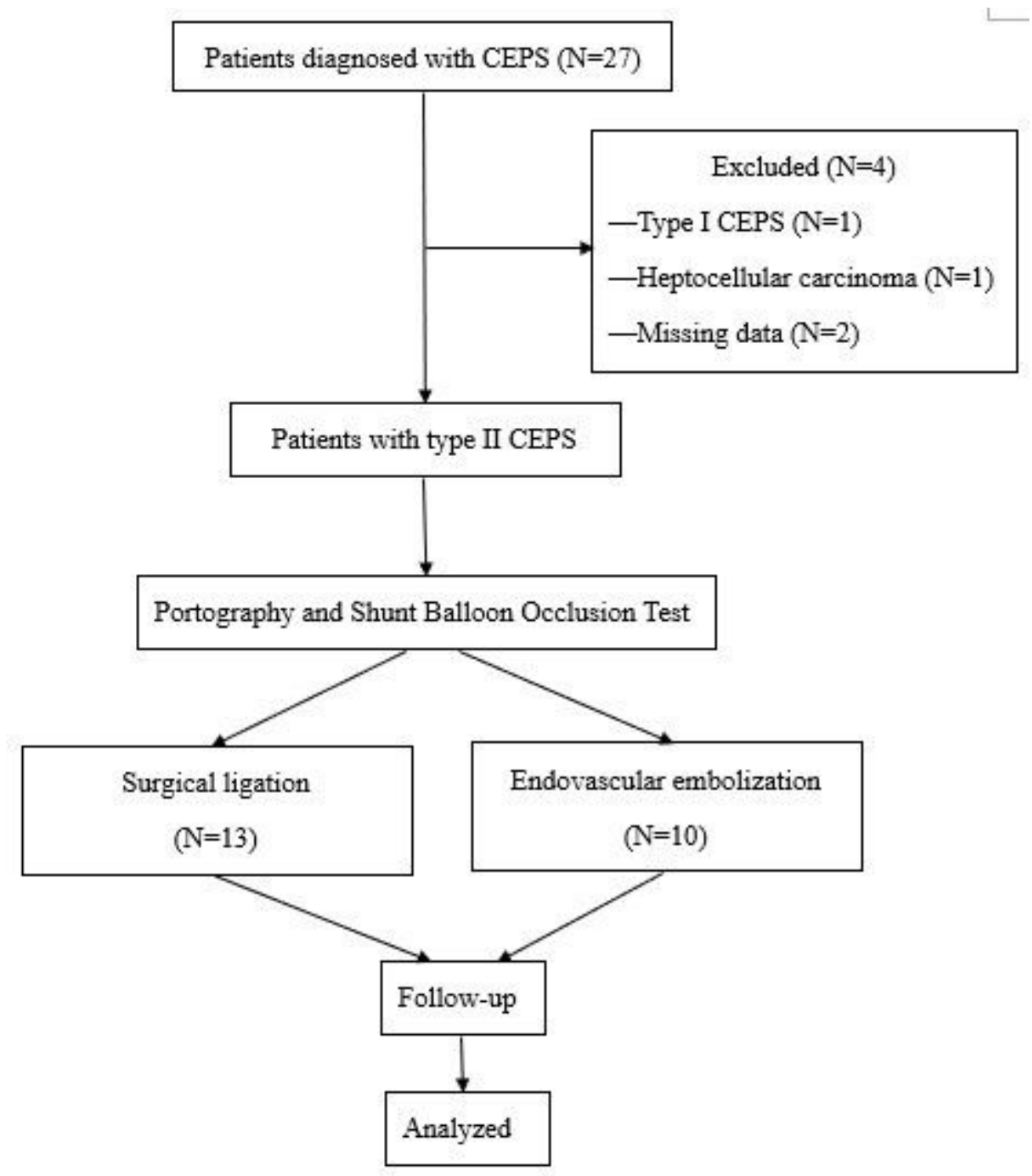

Figure 1 
Flowchart shows study population and groups. CEPS = Congenital extrahepatic portosystemic shunt.
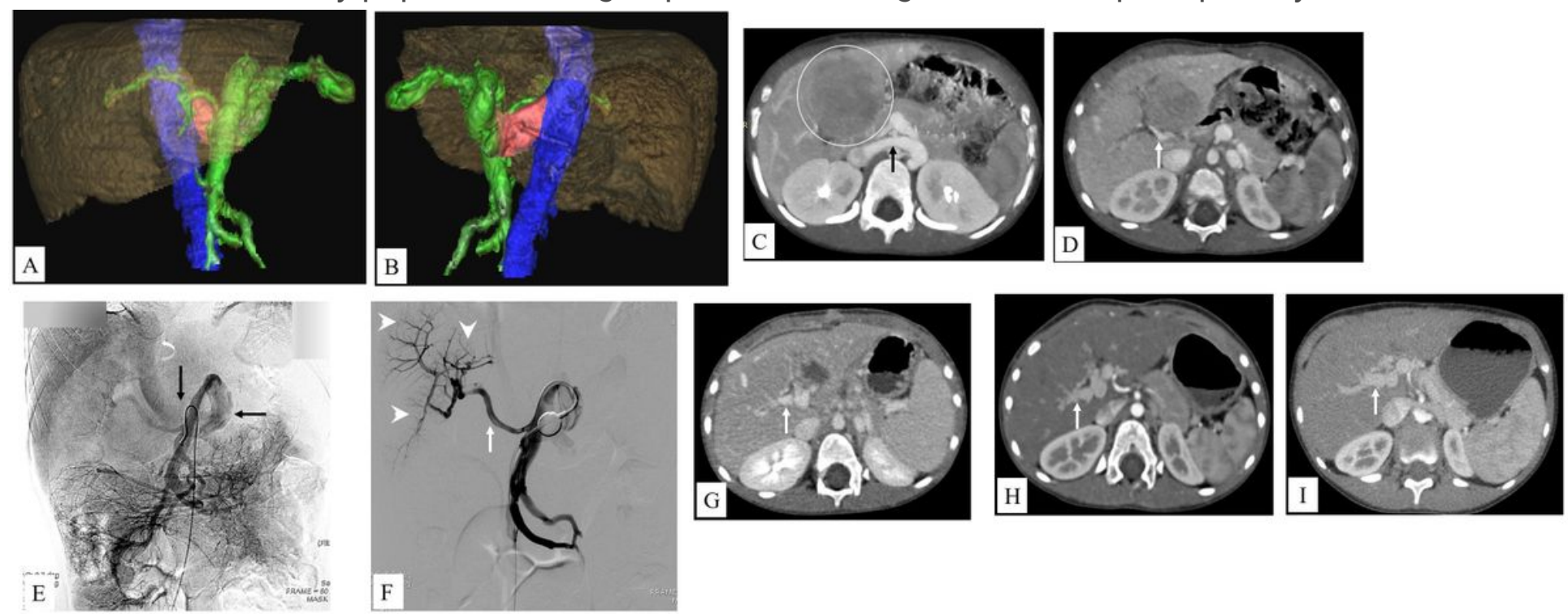

Figure 2

Images in a 3-year old girl diagnosed with type II CEPS with hepatic encephalopathy (HE) underwent surgical ligation due to large portal vein-IVC shunt. (a) Virtual reality (VR) images reconstructed from computed tomographic angiography (CTA) data prior to ligation. Anteroposterior view shows portal vein and its branches (green), inferior vena cava (IVC) (blue) and the patent shunt communicating portal vein and IVC (red). (b) Posteroanterior VR image shows portal vein and its branches (green), IVC (blue) and the portocaval shunt (red). (c) Axial CT image shows the large portal vein-IVC shunt (black arrow) and hepatic adenoma (circle) diagnosed by biopsy. (d) Axial contrast-enhanced CT image shows hypoplastic intrahepatic portal veins (white arrow) preoperetive. (e) Indirect portal venography via superior mesenteric artery (SMA) demonstrates venous outflow of the superior mesenteric vein (SMV) through the shunt (black arrow) drained into IVC (curved arrow), main portal vein (white arrow) and hypoplastic intrahepatic portal vein branches (arrowhead) are visible. (f) Portal venography with balloon occlusion shows fine main portal vein (white arrow) and hypoplastic intrahepatic portal vein branches (arrowhead). The portal venous pressure (PVP) is 14.7 and $16.5 \mathrm{mmHg}$ before and $15 \mathrm{~min}$ after balloon occlusion, respectively. (g) Contrast-enhanced CT images demonstrates the intrahepatic portal veins at 1 month after surgical liagtion. (h) CT scan shows the intrahepatic portal veins at 12 months after surgical liagtion. (i) CT scan shows the intrahepatic portal veins at 60 months after surgical liagtion, the portal vein grows well over time. 

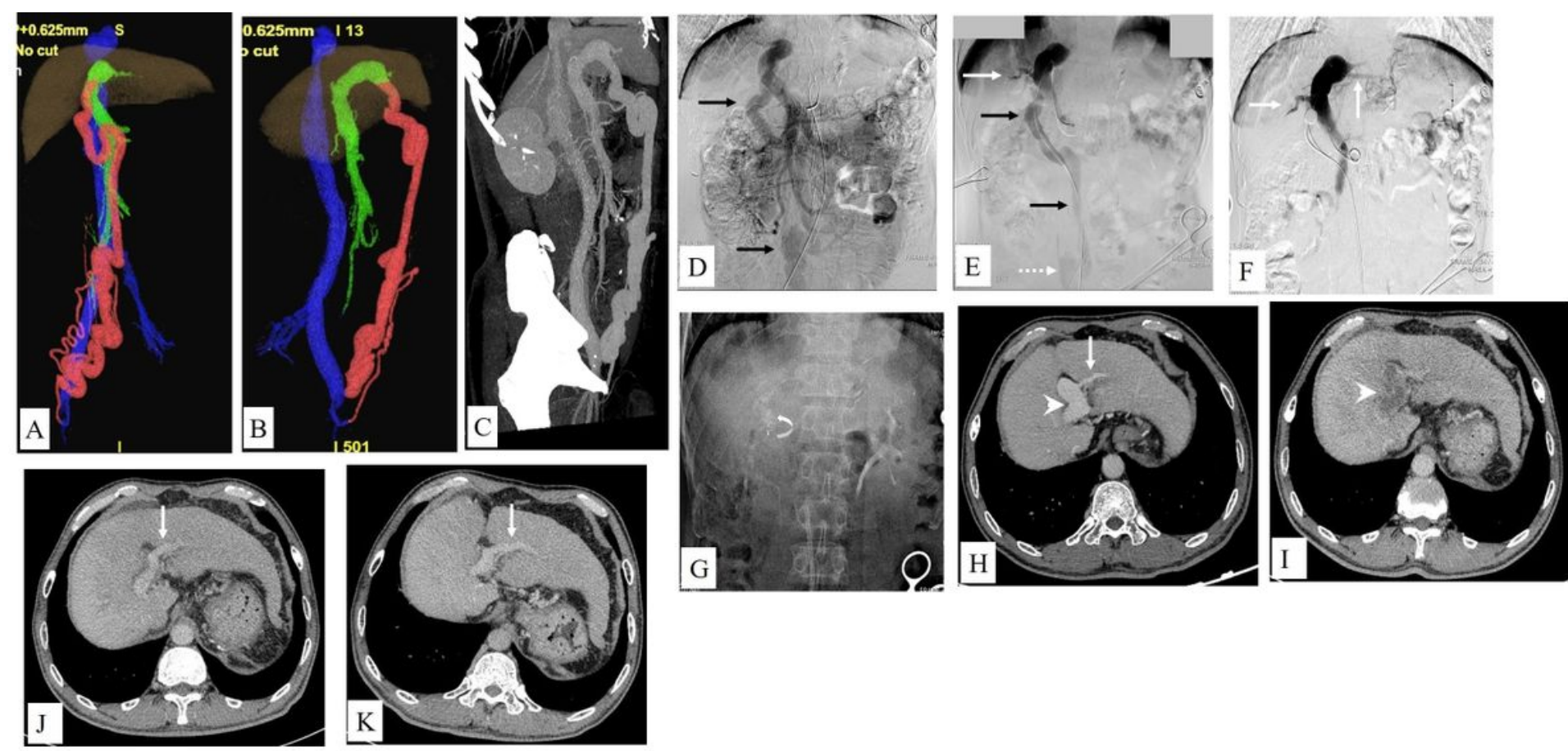

\section{Figure 3}

Images in a 54-year old male diagnosed with type II CEPS with HE underwent endovascular embolization. (a) Anteroposterior VR image reconstructed from CTA data prior to occlusion shows portal vein and its fine intrahepatic branches (green), IVC (blue) and tortuous and dilated portal vein-iliac vein shunt via paraumbilical vein (red). (b) Sagital VR image shows portal vein and its branches (green) IVC (blue) and the shunt (red). (c) Sagital maximum intensity projection at portal venous phase demonstrates the portal vein, IVC and the shunt, in accordance with the VR findings. (d) Indirect portal venography via SMA demonstrates portal venous outflow drained into a tortuous and dilated shunt (black arrow). (e) Portal venography demonstrates partial hypoplastic intrahepatic portal venous veins (white arrow) and the communication between portal vein and right iliac vein (dotted arrow) via the shunt (black arrow). (f) Portal venography with balloon occlusion shows hypoplastic intrahepatic portal veins (white arrow). The PVP is 21.7 and $24.3 \mathrm{mmHg}$ before and $15 \mathrm{~min}$ after balloon occlusion, respectively. (g) The shunt was embolized with Amplatzer plug (curved arrow). (h) Preoperative contrast-enhanced CT image demonstrates the dilated main portal vein (arrowhead) and the hypoplastic intrahepatic portal vein branches (white arrow). (i) CT scan shows portal vein thrombosis (arrowhead) at 1 month after the procedure which disappeared after seven days anticoagulation therapy. (j) Contrast-enhanced CT images demonstrates main portal vein shrinking into normal level (arrowhead) and the intrahepatic portal veins (white arrow) grow well at 12 months after interventional occlusion. (k) CT scan demonstrates the intrahepatic portal veins (white arrow) grow well at 36 months after the procedure. 

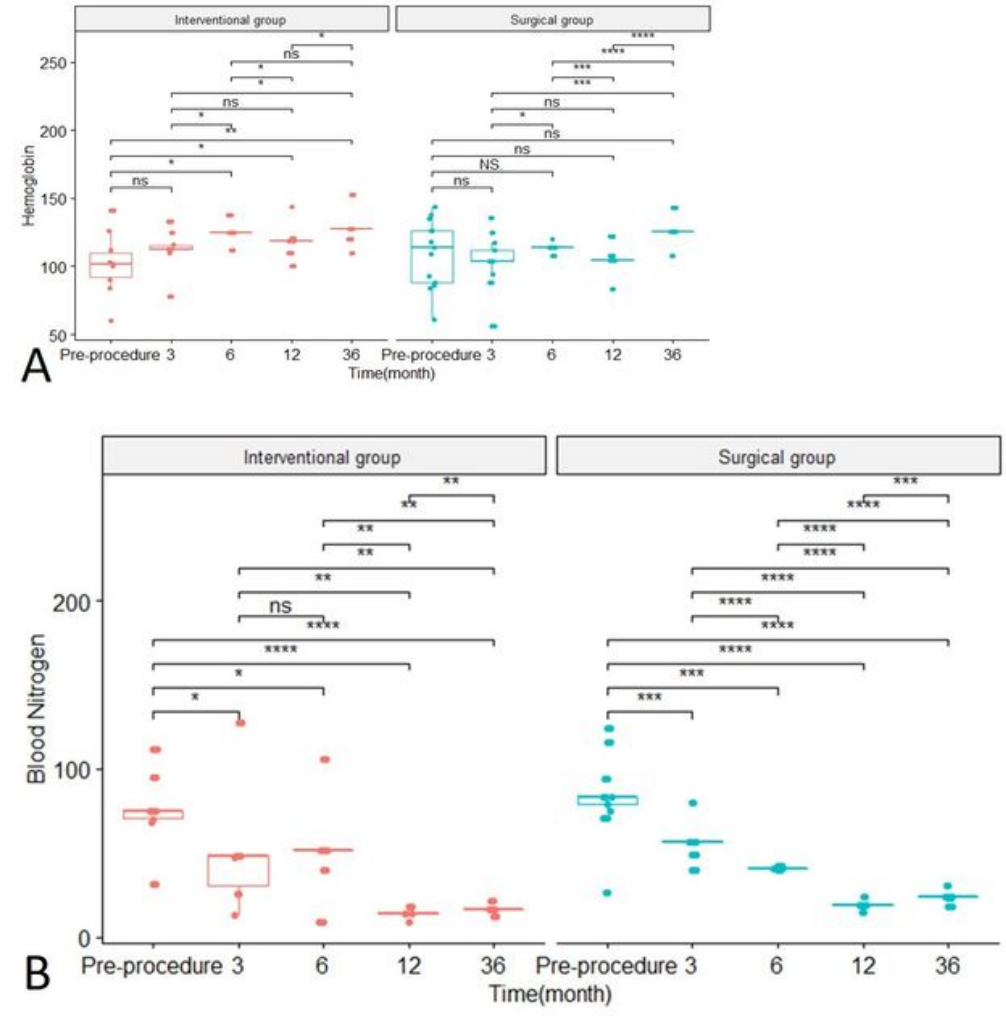
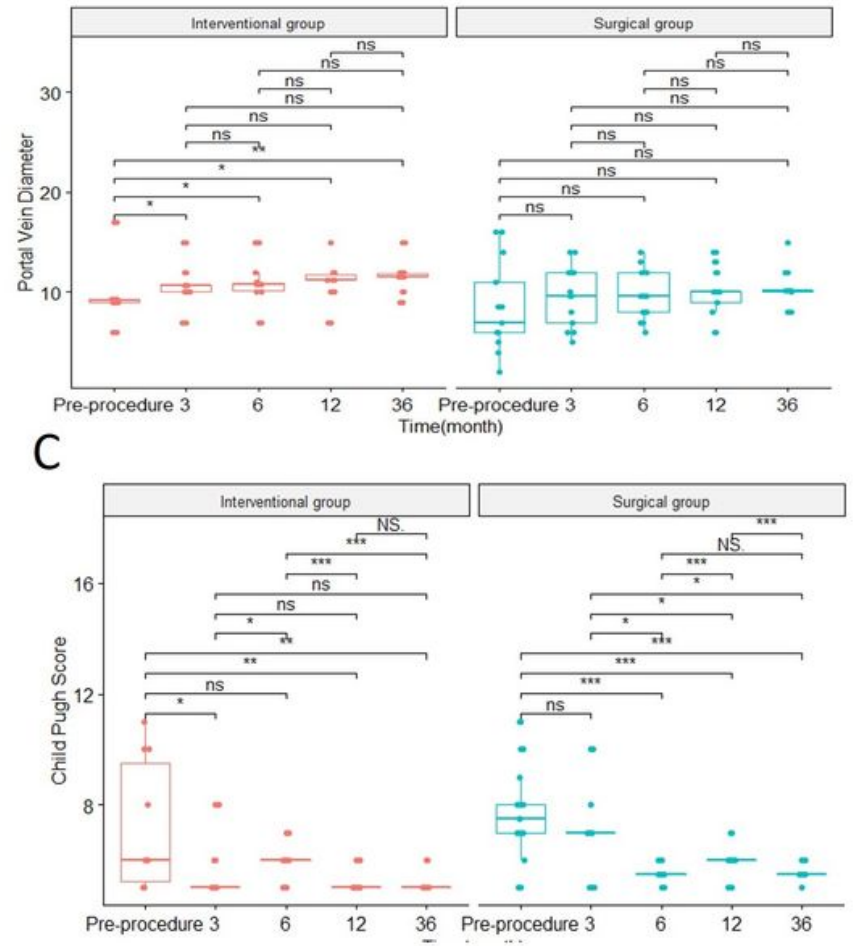

D

\section{Figure 4}

(a) Within-group comparison of hemoglobin in interventional and surgical groups pre- and postprocedure. (b) Within-group comparison of serum ammonia in interventional and surgical groups pre- and post-procedure. (c) Within-group comparison of portal vein pressure in interventional and surgical groups pre- and post-procedure. (d) Within-group comparison of Child Pugh score in interventional and surgical

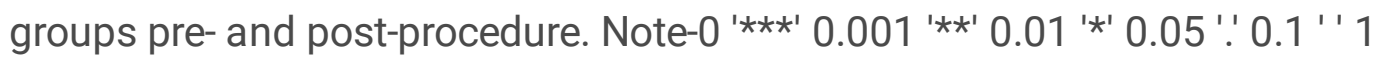

